\title{
The Evolution of Photospheric Source Regions of CMEs
}

\author{
K. Muglach ${ }^{1}$ and K. Dere ${ }^{2}$ \\ ${ }^{1}$ Artep, Inc., Naval Research Laboratory, Washington, DC, USA \\ email: muglach@nrl.navy.mil \\ ${ }^{2}$ George Mason University, Fairfax, VA, USA \\ email:kdere@gmu.edu
}

\begin{abstract}
In this presentation we determine the source regions of CMEs that were observed with SoHO/LASCO during times of solar activity maximum (Feb./Mar. 2000) and during the declining phase of the solar cycle (Nov./Dec. 2002). The CMEs were traced back onto the disk and EIT EUV images were used for identifying the sources. With the help of MDI synoptic magnetograms we follow the evolution of the photospheric magnetic flux about $24 \mathrm{~h}$ before and $12 \mathrm{~h}$ after the event. We find that about $87 \%$ of the identified CME source regions show smallscale flux changes before the event, usually flux emergence and/or flux disappearance. In $13 \%$ of the cases we find no signature of photospheric flux changes.
\end{abstract}

Keywords. Sun: magnetic fields, Sun: photosphere, Sun: coronal mass ejections (CMEs)

\section{Introduction}

The solar magnetic field is thought to play a fundamental role in the process that leads to coronal mass ejections (CMEs). Ideally one would wish to know the complete large-scale 3d structure of the magnetic field involved. Coronal magnetic fields can not be directly measured at the present time although a first successful attempt has recently been carried out by Lin et al. 2004. Coronal fields are usually indirectly inferred e.g. from an extrapolation of the field at the solar surface. In this contribution we study the evolution of the photospheric magnetic field in active regions (ARs) and around filaments that produce CMEs. These photospheric fields represent the lower boundary of the $3 \mathrm{~d}$ structure. Field changes like flux emergence, submergence, reconnection or shearing have been found associated with various solar eruptive phenomena like flares or disappearing filaments. The current work is the continuation of an earlier analysis by Subramanian \& Dere (2001).

\section{Observations}

We examined data of two months of CME events in 2000 (Feb./Mar.) and 2002 (Nov./Dec.) during the peak and the declining phase of the solar cycle. We make use of several SoHO instruments: the LASCO/C2 coronograph, EIT (195 A coronal images) and MDI (longitudinal magnetograms). Event and source detection followed the steps outlined below. We selected events where we could unambiguously determine the source region and study the short-term photospheric magnetic activity related to the CME. E.g. multiple sources were rejected, when several ARs showed coronal signatures at the projected time and location. Sources very near the limb were also rejected as the lineof-sight component of the magnetic field is reduced near the limb and weak field changes 


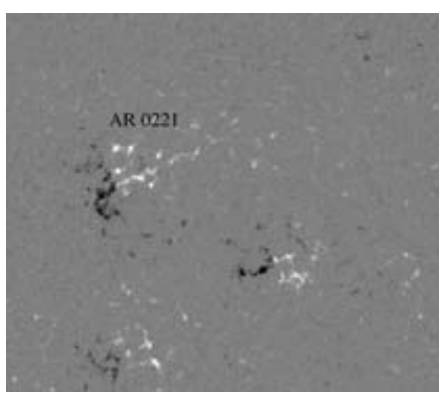

Figure 1. event A, magnetogram taken on $12 \mathrm{th}$ Dec. 2002, 16:00UT.

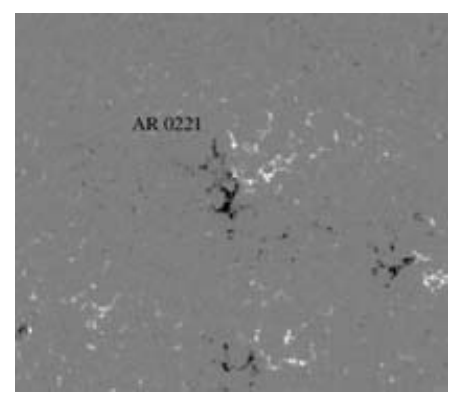

Figure 2. event A, magnetogram taken at 17:41UT, event onset in EIT at 17:36UT, magnetic flux of both polarities disappears in the northern part of AR 0221 .

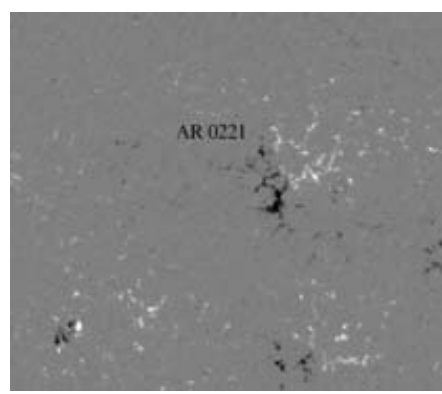

Figure 3. event A, magnetogram taken on 13th Dec. 2002, 04:53UT.

can not be detected (e.g. in filaments channels). In addition flux changes due to solar rotation and due to the evolution of the AR itself can not be disentangled near the limb.

In the first step we used the CME lists and catalog which are available on the LASCO public web-site (http://lasco-www.nrl.navy.mil/cmelist.html). All events in the catalog were checked regardless of their size. Among others the catalog gives position angle and size of the CME and the time of its first signature in $\mathrm{C} 2$. We also compared the list with the daily on-line $\mathrm{C} 2$ movies to identify coronal outflows as CME signatures. These movies have a temporal resolution of about $20 \mathrm{~min}$.

Then we used coronal images in EIT $195 \AA$ to search for possible source regions. These synoptic full-disk images are taken about every $12 \mathrm{~min}$. Various signatures of activity were used like brightenings, dimmings and EIT waves. We allowed for a time difference of up to $2 \mathrm{~h}$ before the first $\mathrm{C} 2$ signature and assumed an approximately radial outflow of the plasma. Dark filament channels were also taken into account, usually in addition to checking $\mathrm{H} \alpha$ images from various public web-sites.

Finally MDI line-of-sight magnetograms were used to investigate photospheric flux changes. We used 96 min cadence full-disk synoptic data and studied a period of about $24 \mathrm{~h}$ before and $12 \mathrm{~h}$ after the event. Some events had to be rejected as there were not sufficient MDI data available.

\section{Results}

Out of several hundred CMEs listed in the catalog we ended up with 32 events with an identified source region. $28(87 \%)$ of them showed changes in the magnetic flux. For four events (13\%) no flux changes could be detected. Figures 1-9 give three examples to demonstrate the variety of flux changes that are present in the data. The magnetogram in Fig. 1 of event A is taken on 12.Dec. 2002 at 16:00UT. The event onset in EIT was on 13.Dec. 2002 at 17:36UT, the magnetogram in Fig. 2 was taken at 17:41UT. In the northern part of AR 0221 patches of opposite polarity flux move closer and disappear as can be seen in Fig. 3 (flux cancellation). Event B in Figs. 4-6 (AR 8904) is a case of flux emergence: in the southern part of the AR white polarity flux appears at the edge of the black polarity flux of the bipolar region. This flux emergence continues after the CME for many more hours (although it is eventually masked by solar rotation effects) and the AR has two more CMEs in the following 20h. Finally, the flux changes in event 


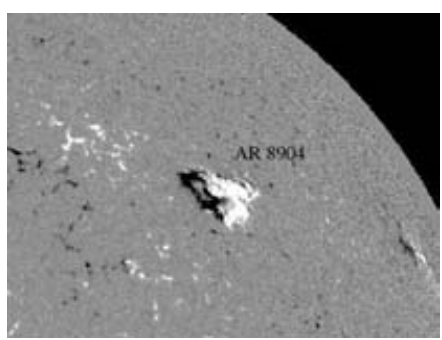

Figure 4. event B, magnetogram taken on 10th Mar. 2000, 23:59UT.

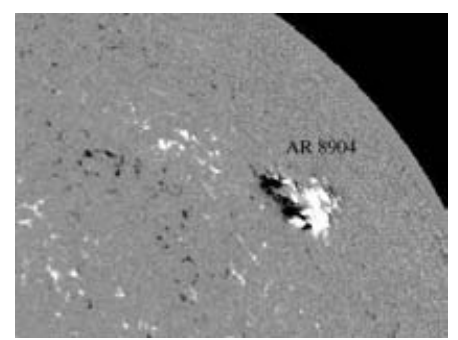

Figure 5. event B, magnetogram taken on 11th Mar. 2000, 20:47UT, event onset in EIT at 21:23UT, white polarity flux emerges in the southern part of AR 9804, flux emergence continues over many hours after the event and the AR shows two more CMEs.

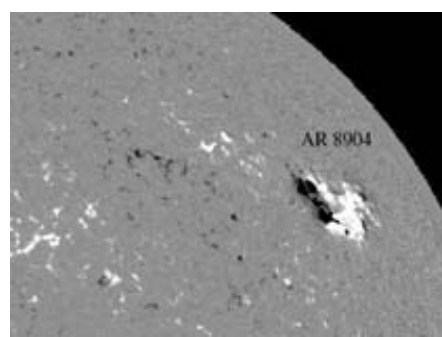

Figure 6. event B, magnetogram taken on 12th Mar. 2000, 09:41UT, flux emergence seems to continue after the first event but the AR gets too near to the limb and solar rotation masks actual flux changes.

$\mathrm{C}$ in Figs. 7-9 are rather complex: in the northern part we can see flux emerging (white, Fig. 8) and at the same time the main white polarity structure gets weaker and decays. The decay continues after the CME (Fig. 9).

From the 28 events that showed flux changes we can summarize the following points:

- Flux changes are all small-scale. They can be the size of a small dipole (a few pixels in MDI) up to the changes seen in event B (Figs. 4-6).

- Flux changes happen several hours before the CME onset, some continue after the event as can be seen in the examples given here.

- Flux emergence always seems to happen in an already existing AR. There are several cases of flux emergence in the MDI data where a new AR is formed away from any preexisting AR. This leads to enhanced coronal activity (as seen in EIT) but not to a CME.

- Flux changes happen right at or very near the onset location as determined by EIT.

\section{Discussion}

Subramanian \& Dere (2001) surveyed 32 CMEs during the rising phase of the solar cycle. They also found that flux emergence and cancellation are associated with the CMEs. The flux changes happen in time-scales of several hours. The initiation of the events happens much faster, probably faster than the cadence of EIT (12 min) which we can also confirm from our current data set. Thus, we consider the flux changes as a trigger of the CME.

Flux emergence in connection with CMEs has been noted before by several authors, like Feynman \& Martin (1995), Wang \& Sheeley (1999) and Chen \& Shibata (2000) for CMEs due to filament eruptions, and Lara et al. (2000) for flaring ARs.

Note that with the line-of-sight magnetograms we can only describe the flux changes that can happen during CME events (e.g. Wang, 2001). To provide a physical interpretation we would need to know the full magnetic field vector, preferably derived from full Stokes spectro-polarimetry. E.g. if flux cancellation is associated with the submergence of a flux tube which connects the two polarities, then this would have clear signatures in the magnetic field vector (e.g. in an increase of the horizontal component of the field). Recent spectro-polarimetric observations of flux cancellation indicate such a scenario (Chae et al. 2004). Also for a proper correction of solar rotation the magnetic field 


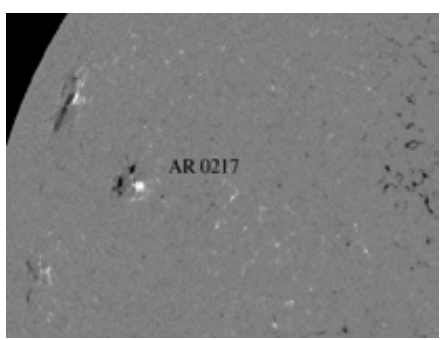

Figure 7. event C, magnetogram taken on 9th Dec. 2002, 12:48UT.

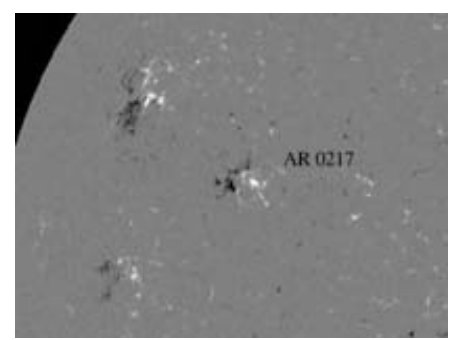

Figure 8. event C, magnetogram taken on 10th Dec. 2002, 16:05UT, event onset in EIT at 15:48UT, flux changes are complex, white polarity flux emerges in the northern part of AR 0217, while the central flux region decays (seen also Fig.9).

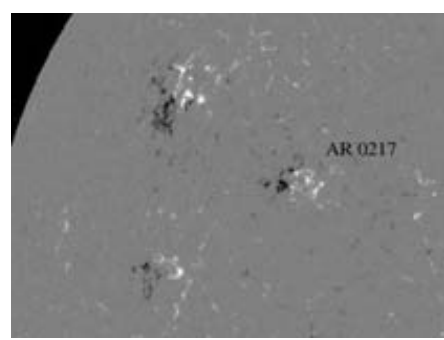

Figure 9. event C, magnetogram taken on 11th Dec. 2002, 04:53UT.

vector is essential, as the assumption of a radial magnetic field is often not valid, e.g. in a sunspot penumbra and in case of flux emergence or retraction.

This study would also benefit a lot from better resolution, spatial resolution and in particular temporal resolution. Although the flux changes happen over several hours the 96 min synoptic data cover that period only roughly. In some cases 1 min cadence MDI magnetograms were available and five minute averages produced the best magnetograms but usually they did not cover a period of $24 \mathrm{~h}$ before the event. The up-coming Solar-B mission will have the ability to provide this kind of data.

\section{Acknowledgements}

K.M. acknowledges financial support from the NRL LASCO project The SoHO data used in this work are courtesy of SoHO/LASCO, SoHO/EIT and SoHO/MDI consortium. $\mathrm{SoHO}$ is a project of international cooperation between ESA and NASA.

\section{References}

Chae, J., Moon, Y-J., \& Pevtsov, A.,A., 2004 ApJ 602, L65

Chen, P.F., Shibata, K. 2000, ApJ 545, 524

Feynman, J., \& Martin, S.F. 1995, JGR 100, 3355

Lara, A., Gopalswamy, N., \& DeForest, C. 2000, GRL 27, 1435

Lin, H., Kuhn, J.R., \& Coulter, R. 2004 ApJ 613, L177

Subramanian, P., \& Dere, K.P. 2001, ApJ 561, 372

Wang, J. 2001, SSR 95, 55

Wang, Y.-M., \& Sheeley, N.R. 1999, ApJ 510, L157

\section{Discussion}

Gopalswamy: You have classified AR8904 as a case of flux emergence. However, at the same location where the white polarity emerges, there is black polarity disappearing. So, the classification of this must be "complex" rather than emergence or cancellation.

MugLACH: You are correct, some of the black polarity flux disappears. But the increase of white polarity flux clearly dominates, so we have classified this event as flux emergence. In event $\mathrm{C}$ (complex) some of the white polarity flux seems to emerge next to the black 
one and the main white polarity flux decays. But it might also be that the flux that seems to dispersed flux from the decaying region. The current $96 \mathrm{~min}$ MDI data does not allow one to differentiate between these two possibilities. I also do not want to overemphasize (sub) categories here. We just want to show the variety of flux changes in our sample of source regions.

Jun LIN: Corresponding to these features observed in EIT \& MDI, what does the evolution in those observed in $\mathrm{H} \alpha$ look like?

MuglaCH: We have checked $\mathrm{H} \alpha$ data for some events where a filament eruption seemed to be involved. This was primarily done to verify that an $\mathrm{H} \alpha$ filament was involved. A few co-temporal $\mathrm{H} \alpha$ movies confirmed the filament eruption, in some other cases the pre-event filament had disappeared (in images taken several hours before and after the event).

Kоштснму: You are talking about changes of the "flux" of the longitudinal component of the magnetic field, not the changes of the magnetic fluxes. Did you look at the possibility to explain your observations by assuming only change of the direction (topology) of the field?

MuglaCH: MDI measures magnetic flux, a combination of $|\vec{B}|, \cos \phi$ and $\alpha(\phi=$ angle between the line of sight and $\vec{B}, \alpha$ magnetic filling factor). Flux changes can be due to any one of these three or a combination of them. We avoided events too close to the limb as they are dominated by line-of-sight effects. Part of the observed flux changes are nevertheless probably due to changes in $\cos \phi$ : e.g., in event A flux disappears by flux cancellation. If we assume a small loop connecting the two polarities submerges, then this would be an example of flux changes due to topological changes. To determine the physical process involved in the flux changes one needs the vector field $\vec{B}$, ideally derived from spectro-polarimetry which can determine $\vec{B}$ and $\alpha$.

VOURLIDAS: Flux emergence occurs always on the solar photosphere. How can we be sure that your statistics are not just a coincidence?

Muglach: The fact that these flux changes are almost always at the onset location makes me think that they are not random. In the case of flux emergence, a pre-existing large-scale magnetic structure seems also to be necessary. I have seen several cases of flux emergence in the quiet sun (away from active regions) that did not result in a CME (within 24 hours). Thus, flux emergence seems to be a necessary but not sufficient condition for a CME.

Nindos: Comment: Sometimes flux emergence occurs not only within an old AR but also in quiet sun regions resulting in CME-productive ARs.

MugLACH: I can only comment on the sample I have currently available, and I did not see this.

SCHWEnN: Did you measure the emerging flux quantitatively? MDI allows that?

Muglach: No, I did not do any quantitative calculations. To do this, I have to correct for projection effects and solar rotation. Accurate correction can only be carried out when having the vector field available. E.g., the assumption of a radial field that is often used is not valid in a sunspot penumbra and many of the active regions in the sample have a complex flux distribution. 